\title{
Natur und ... woran Sie jetzt noch denken
}

Andreas Losch

Das könnte allerdings vieles sein, dieses, was man der Natur gegenüberstellt oder mit ihr verbindet. Gegenbegriffe sind Kultur, Geist oder Gnade, aber auch die Technik, die wiederum oft in Gegensatz zu dem so schön klingenden Paar "Natur und Umwelt" gestellt wird. Das Denken selbst aber vollzieht sich mit dem Geist, und dem Bedenken dieses Paares "Natur und Geist« wollen wir uns hier widmen.

Wir sind sicher nicht die ersten, die das tun. Wenn man allein die veröffentlichten Bücher berücksichtigt, gibt es sehr reichhaltige Literatur, auch in jüngster Zeit; da verengt sich der Geist dann gern aufs Gehirn ${ }^{1}$ - anders als bei Viktor von Weizsäcker, seines Zeichens Arzt und Philosoph, manchen gilt er als Begründer der Psychosomatik, also gerade der Aufzeigung des Zusammenhangs zwischen Geist und Körper. ${ }^{2}$

Viktor von Weizsäcker hat über Natur und Geist früh nachgedacht. Unter diesem Titel,»Natur und Geist«, hat er seine "Erinnerungen eines Arztes« veröffentlicht. ${ }^{3}$ Viktor von Weizsäcker war es wichtig, einen Zusammenhang von Natur und Geist zu sehen; gegen den cartesischen Dualismus von "res cogitans« und "res extensa betonte er das Grundverhältnis zur Natur, in dem wir Menschen stehen, und die Idee der Kohärenz im "Gestaltkreis «" ${ }^{4}$ seinem sicher einflussreichsten Werk; es wurde als Überwindung des psychophysischen Dualismus gelobt, hatte anfänglich aber deswegen auch schwer zu kämpfen. ${ }^{5}$

Worum geht es bei der Idee des Gestaltkreises? Es ist eine Theorie der Einheit von Wahrnehmung und Bewegung, wie wir sie in der Verschmelzung von unserem Denken und Fühlen mit unserer Umwelt in unseren alläglichen Handlungen beobachten und reflektieren können.Viktor von Weizsäcker bringt verschiedene Beispiele hierfür: unseren unbewussten Umgang mit unserem Hausschlüssel

\footnotetext{
1 Eine differenzierte Behandlung der Bewusstseinsfrage findet sich jetzt bei HansDieter Mutschler, Bewusstsein. Was ist das?, Leipzig 2018.

2 Der ehemalige deutsche Bundespräsident Richard von Weizsäcker, wie auch der bekannte Physiker und Philosoph Carl Friedrich von Weizsäcker waren seine Neffen. Zu Biografischem s. Udo Benzenhöfer, Der Arztphilosoph Viktor von Weizsäcker. Leben und Werk im Überblick, Göttingen 2007.

3 Vgl.Viktor von Weizsäcker, Natur und Geist [1964], München 2004.

4 Viktor von Weizsäcker, Der Gestaltkreis, Stuttgart ${ }^{6} 1996$.

5 Vgl.Viktor von Weizsäcker, Natur und Geist [1964], München 2004, 62.
} 
oder Kragenknopf, oder auch das Hantieren mit einem Gummiball. In der Analyse jedweder solcher Vorgänge wird jeweils deutlich, dass weder der über die zentrifugalen Nerven stattfindende Bewegungsvorgang noch der über die zentripetalen Nerven stattfindende Wahrnehmungsvorgang weggelassen werden kann, ohne dass die vollzogene Handlung oder Leistung sofort zerbricht. ${ }^{6}$

Der Zusammenhang lässt sich am besten kreisförmig darstellen, woraus sich der Grundgedanke der Kohärenz ergibt. Z.B. bewegen sich die Schenkel eines Reiters kohärent mit den Bewegungen des Pferdes, ohne das es dafür eines Steuerungsimpulses bedarf; "ein Reiz ist in diesem Kreisschema nicht positiv als neuer Impuls zu verstehen, sondern negativ als Aufhebung der Kohärenz«?

Viktor von Weizsäcker ordnet die Kräfte der Kohärenz auf zwei Mittelpunkte hin zu, die er als I(ch)-Kern und als U(mwelt)-Kern bezeichnet, wobei deren Grenzen flexibel zu denken sind. Die Frage also "wo mein leiblicher und seelischer Besitz beginne und meine Umwelt aufhöre", ist "nicht an Gegebenheiten der Erscheinung abzulesen ${ }^{8}$. Dies erinnert an eine Schlüsselerfahrung Viktor von Weizsäckers im Krieg, »in welchem sich mir die ursprüngliche Ungeschiedenheit von Subjekt und Objekt gleichsam leiblich denkend offenbart hat. Bei ruhigem Betrachten einer dort hängenden Patronentasche bin ich Patronentasche, und diese ist ich."

Kann man diese "Offenbarungserfahrung" auch verobjektivierend als dehydriertes Delirium auf dem Felde deuten, lässt sich dennoch annehmen, dass sie Viktor von Weizsäckers spätere Forschungen und den Gedanken der Kohärenz motiviert hat. Auch wenn die Annahme einer Einheit von Ich und Umwelt zunächst sehr weit zu gehen scheint, sind ihre Grenzen in der Tat verschiebbar. Für ein Individuum, das nacheinander "isst, schläft, spielt, zeugt, kämpft» ist die Grenze zwischen ihm und seiner Umwelt jeweils anders verortet. "Es ist nicht immer dasselbe, und seine Umwelt ist nicht immer dieselbe, wenn es seine Tätigkeit auf so eindrucksvolle Weise ändert. «10

Die Verschmelzung von Individuum und Umgebung wurde, was den Gebrauch von Werkzeugen angeht, auch von anderen Philoso-

\footnotetext{
6 Vgl. Andreas Losch, Jenseits der Konflikte. Eine konstruktiv-kritische Auseinandersetzung von Theologie und Naturwissenschaft, Göttingen 2011, 218. Als herausgehobenstes Beispiel erscheint beiViktor von Weizsäcker der Nystagmus, das Augenzittern beim Schwindel, hierzu hat er ein eigenes Experiment erstellt.

7 Ebd.

8 Weizsäcker, Der Gestaltkreis, dargestellt als psychophysiologische Analyse des optischen Drehversuchs, in: Pflügers Archiv 231 (1933), 655.

9 Ders., Natur und Geist, 68.

${ }^{10}$ Ders., Der Gestaltkreis, 656.
} 
phen postuliert und beobachtet. Der ungarische Wissenschaftsphilosoph Michael Polanyi z.B. weist darauf hin, wie diese in die eigenen Handlungsabläufe inkorporiert werden. "Denken wir daran, wie ein Blinder seinen Weg mittels eines Stocks erfühlt; dies involviert die Umwandlung der Stöße, die an seine Hand and an die den Stock haltenden Muskeln weitergegeben werden, in eine Wahrnehmung der Dinge, die von der Spitze des Stocks berührt werden. " $^{11}$ "Wie» und "Was" gewusst wird, sind in ihrer Struktur hier offensichtlich einander sehr ähnlich, wie Polanyi betont. ${ }^{12}$ Ich denke, man könnte auch Viktor von Weizsäckers These der Einheit von Wahrnehmung und Bewegung durch das Beispiel bestätigt sehen.

Wie verhalten sich also nun Natur und Geist? Wenn Wahrnehmung und Bewegung mit der Umwelt vereint werden, sind Subjekt und Objekt nicht mehr strikt zu trennen, eine Einsicht, die selbst die so um Objektivität bemühte Naturwissenschaft in Form der Reflexion der Quantenphysik zu realisieren beginnt, denn auch hier bedarf es Werkzeuge und Instrumente, um das Vorhandene zu erfassen, und die Einwirkung dieser Instrumente auf das zu Untersuchende erzeugt erst, verändert aber auch, das Ergebnis. Daher ist es nur möglich, Ort oder Impuls eines Elementarteilchens zu bestimmen, nicht aber beides: die berühmte Heisenbergsche Unschärferelation.

Umgekehrt ist dann aber auch an die losgelöste Selbstbestimmtheit unseres Geistes ein Fragezeichen zu setzen. Die Grandiosität der Ichperspektive, untermauert von Descartes Gedanken des "Ich denke, also bin ich « beginnt zu verschwimmen. Was ist die Besonderheit des Menschen? Das er als Ich auftritt? Ist dies das Proprium des Geistes, das Selbstreflexionsvermögen?

Während Viktor von Weizsäcker den cartesischen Dualismus im Gestaltkreis gewissermassen aufhebt, setzt sich Martin Buber auf andere Weise mit der Vorherrschaft des cartesischen Denkens auseinander. Zum »ego cogito, ergo sum« bemerkt er:

»Descartes versucht somit auf dem Wege der Abstraktion die Konkretheit der Ausgangssituation als Erkenntnis zu gewinnen, aber vergeblich. Das Ich der lebendigen Person läßt sich nie in solch einer Ableitung, wohl aber im echten Verkehr mit einem Du als existent erfahren. Auf dem Weg der philosophischen Abs-

\footnotetext{
${ }_{11}$ Michael Polanyi, Personal knowledge. Towards a post-critical philosophy [1958], London 1998, 55f, dt. nach Losch, Jenseits der Konflikte, 170.

12 Michael Polanyi ist besonders für seine Idee unseres stillschweigenden oder impliziten Wissens bekannt.
} 
traktion ist die Konkretheit, von der alles Philosophieren ausgeht, nicht wieder $\mathrm{zu}$ erreichen: sie ist aufgehoben. $\ll^{13}$

Wie Viktor von Weizsäcker setzt auch Buber eine ursprüngliche Bezogenheit des Subjekt-Ichs auf seine Umwelt voraus, versteht diese Bezogenheit aber als von durchaus verschiedener Qualität. Geistreich ist sie nur da, wo sie zwischen einem Ich und einem Du als Beziehung zu Tage tritt, während sie in der Regel aber in einem Ich-Es Verhältnis bleibt. Die Frage bleibt: Woher kommt dabei der Geist, zu dessen »Wiederbringer und Wahrer» der Mensch bestellt $\operatorname{sei}^{14}$, wie entsteht die Erfahrung einer Beziehung, zum Du?

Bubers Antwort ist so schlicht wie überzeugend: aus der Begegnung, die das menschliche Leben von Anfang an prägt. »Im Anfang ist die Beziehung ${ }^{15}$ bezieht sich nicht nur, aber auch auf diese Frage. Buber verfolgt in "Ich und Du« die Entwicklung des Kindes aus der Ich-Du Beziehung heraus. »Daß die geistige Realität der Grundworte sich aus einer naturhaften erhebt, die des Grundworts Ich-Du aus der naturhaften Verbundenheit, die des Grundworts Ich-Es aus der naturhaften Abgehobenheit, wird uns hier unverschleiert klar. ${ }^{16}$

Natürlich kann sich bei Martin Buber die Ich-Du Beziehung auch auf einen Baum erstrecken. Bereits Bubers Frühwerk »Daniel« schildert eingangs eine Naturerfahrung mit einem solchen Gewächs:

"Nach einem Abstieg, zu dem ich ohne Rast das Spätlicht eines vergehenden Tages hatte nutzen müssen, stand ich am Rande einer Wiese, nun des sicheren Weges gewiß, und ließ die Dämmerung auf mich niederkommen. Unbedürftig einer Stütze und doch willens, meinem Verweilen eine Bindung zu gewähren, drückte ich meinen Stab gegen einen Eschenstamm. Da fühlte

\footnotetext{
${ }_{13}$ Martin Buber, Schriften zu Philosophie und Religion, in: MBW 12 (2017), 384.

14 „Der Mensch, das jüngste Kind eines jungen Planeten, zum Wiederbringer und Wahrer des Geistes im Weltall bestellt", so beginnt Buber seinen ersten Entwurf des Anfangs von "Ich und Du«. (Martin Buber Archiv Jerusalem Ms. Arc.Var. 350, Mappe 9a, 97) Aus der Erschütterung der Erfahrung des Todes eines engen Freundes, Gustav Landauers, ist dieser Entwurf hervorgegangen. In "Ich und Du« lebt dessen Geist sozusagen weiter. Siehe Andreas Losch, Überlieferung und Kompositionsstruktur von Ich und Du. Ein Neuansatz der Interpretation, in: ZfBeg 1/2( 2018) 21-35.

${ }^{15}$ Martin Buber, Schriften über das dialogische Prinzip, in: MBW 4 (2019), 48, 53.

${ }^{16}$ Ebd., 52. Franz Rosenzweig hat Buber dafür kritisiert, dass er dem Ich-Du mit seinem Verständnis des Ich-Es »einen Krüppel zum Gegner gibt (Franz Rosenzweig, Der Mensch und sein Werk. Gesammelte Schriften 1. Briefe und Tagebücher 2. Band 1918-1929, 824). In Rosenzweigs eigenem Entwurf, dem "Stern der Erlösung", ist die dritte Person wertgeschätzt als geschaffene Welt.Vgl. Andreas Losch, Ich, Du und ER. Franz Rosenzweigs Einfluss auf die Abfassung von Ich und Du, in: Im Gespräch 16 (2015), 19-35.
} 
ich zwiefach meine Berührung des Wesens: hier, wo ich das Holz hielt, und dort, wo es die Rinde traf. Scheinbar nur bei mir, fand ich dennoch dort, wo ich den Baum fand, mich selber. ${ }^{17}$

Ist hier noch Selbsterfahrung das Ergebnis des frühen Buber, betont der von seiner Zelebrierung jeder mystischen Entwerdung ${ }^{18}$ geläuterte Buber in "Ich und Du« dann drei Erlebnis-Sphären, »in denen sich die Welt der Beziehung errichtet«. In dieser Beziehungswelt ist nun nicht nur das Leben mit Menschen oder gar mit geistigen Wesenheiten, sondern eben auch mit der Natur vorgestellt. ${ }^{19}$

Buber expliziert dies wieder an einem Baum.»Ich kann ihn als Bild aufnehmen" oder "als Bewegung verspüren ${ }^{20}$. »Ich kann ihn einer Gattung einreihen und als Exemplar beobachten, auf Bau und Lebensweise. Ich kann seine Diesmaligkeit und Geformtheit so hart überwinden, daß ich ihn nur noch als Ausdruck des Gesetzes erkenne « ${ }^{21}$. ॥Ich kann ihn zur Zahl, zum reinen Zahlenverhältnis verflüchtigen und verewigen. $\aleph^{22}$ In all dem bleibt der Baum jedoch reiner Gegenstand. Es könne aber auch geschehen, "aus Willen und Gnade in einem", dass ich, den Baum betrachtend, in die Beziehung zu ihm eingefaßt werde, und nun ist er kein Es mehr. ${ }^{23}$ Alles, Bild und Bewegung, Gattung und Exemplar, Gesetz und Zahl, ist dann ununterscheidbar vereinigt.

"Alles, was dem Baum zugehört, ist mit darin, seine Form und seine Mechanik, seine Farben und seine Chemie, seine Unterredung mit den Elementen und seine Unterredung mit den Gestirnen, und alles in einer Ganzheit. Kein Eindruck ist der Baum, kein Spiel meiner Vorstellung, kein Stimmungswert, sondern er leibt mir gegenüber und hat mit mir zu schaffen, wie ich mit ihm - nur anders. $\mathbb{2}^{24}$

Beziehung ist Gegenseitigkeit, das wird daran deutlich, und auch die Natur kann zum Gegenüber werden, wie das Beispiel

\footnotetext{
${ }^{17}$ Martin Buber, Frühe kulturkritische und philosophische Schriften 1891-1924, in: MBW 1 (2001), 183.

${ }^{18}$ Vgl. Ders., Schriften über das dialogische Prinzip, 87ff.

${ }^{19}$ Ebd., 41, 98.

${ }^{20}$ Ebd., 41.

${ }^{21}$ Ebd., 42.

${ }^{22}$ Ebd.

${ }^{23}$ Ebd.

${ }^{24}$ Ebd.
} 
des Baumes zeigt. ${ }^{25}$ Auch hier wurde also beobachtet, wie die Grenzen von Ich und Umwelt verschwimmen können, ohne jedoch in einer mystischen Einheitserfahrung zu verschwinden. In diesen anticartesischen Entwürfen sind Natur und Geist niemals Gegenbegriffe, sondern Geist wird aus der Natur geboren.

Während Descartes an das "ego cogito, ergo sum" anschliesst, "daß ich eine Substanz bin, deren ganzes Wesen oder deren Natur darin besteht, zu denken und die zum Sein keines Ortes bedarf, noch von irgendeinem materiellen Dinge abhängt«, so versteht er die Seele ${ }^{26}$, ist das losgelöste Ich bei Buber keiner Rede wert. Geist ist nicht im Ich oder im Du (was ja - wenn überhaupt - auch nur ein anderes Ich ist), sondern zwischen Ich und Du zu finden. ${ }^{27}$ Geist ist sozusagen ein Beziehungsgeflecht. Vielleicht ist es Liebe.

Dies gilt auch für den grössten aller Geister, Gott. Während Descartes aus der Erkenntnis der Beschränktheit seines Denkvermögens auf ein vollkommeneres Wesen schliesst, von dem er abhänge und dies als Gott bezeichnet, ${ }^{28}$ ein noch grandioseres Ich könnte man sagen, so ist Gott für Buber das »ewige Du«, wie es sich in der Hebräischen Bibel offenbart. ${ }^{29}$

Für die christliche Dogmatik ist Gott ja in sich selbst Beziehung. Bedarf dagegen »der Gott Bubers" der Beziehung zum Menschen? Schauen wir uns an, wie Buber das "ewige Du« charakterisiert.

"Die verlängerten Linien der Beziehungen schneiden sich im ewigen Du. Jedes geeinzelte Du ist ein Durchblick zu ihm. Durch jedes geeinzelte Du spricht das Grundwort das ewige an. Aus diesem Mittlertum des Du aller Wesen kommt die Erfülltheit der Beziehungen zu ihnen, und die Unerfuilltheit. Das eingeborene Du verwirklicht sich an jeder und vollendet sich an

\footnotetext{
25 "Mir begegnet keine Seele des Baums und keine Dryade, sondern er selber«, ebd., 42. Es stellt sich die Frage, wenn doch der Baum auch ein Du darstellen kann, ob auch jedes Du ein Ich ist oder werden kann. Vgl. dazu Bubers späteres Nachwort zu Ich und $D u$, jetzt in: Schriften über das dialogische Prinzip, 243-251, insbesondere 244f.

${ }^{26}$ René Descartes, Von der Methode des richtigen Vernunftgebrauchs und der wissenschaftlichen Forschung: Französisch-deutsch, in: [1960] Hamburg ${ }^{2} 1997,55$.

27 "Geist ist nicht im Ich, sondern zwischen Ich und Du. Er ist nicht wie das Blut, das in dir kreist, sondern wie die Luft, in der du atmest. Der Mensch lebt im Geist, wenn er seinem Du zu antworten vermag. Er vermag es, wenn er in die Beziehung mit seinem ganzen Wesen eintritt.Vermöge seiner Beziehungskraft allein vermag der Mensch im Geist zu leben." Buber, Schriften über das dialogische Prinzip, 59.

${ }^{28}$ Vgl. Descartes, Von der Methode, 55. 57.

${ }^{29}$ Vgl. Martin Buber, Schriften zur biblischen Religion, in: MBW 13 (2019).
} 
keiner. Es vollendet sich einzig in der unmittelbaren Beziehung zu dem Du, das seinem Wesen nach nicht Es werden kann. «

Es erscheint hier nicht so, dass Geist aus den Beziehungen hervorgeht oder gar aus der Natur emergiert, vielmehr korrespondiert sozusagen die Ich-Du Beziehung dem ewigen Du, partizipiert der Einzelne in seiner Du-Bezogenheit an einer vorgefunden Du-Wirklichkeit, wie er atmet und im Licht der Sonne wandelt. ${ }^{31}$ Bubers Gottesbegriff ist demnach durchaus transzendent zu denken, in einer Weise, die in dem unverfügbaren Aufscheinen der Du-Beziehung transparent und immanent wird. ${ }^{32}$ Das ist dann Gnade.

Was sind wir also? Was ist Geist? Natur, die ihrer selbst bewusst wird? Populär ausgedruckt gilt: "Wir sind der Teil des Kosmos, der sich nach sich selbst fragt. ${ }^{33}$ Als solcher sind wir jedoch in der Lage zu erkennen, dass wir auf Gnade angewiesen sind, um unsere Besonderheit geistvoll zu leben. Und davon, wie wir unsere Umwelt durch unsere Technik inkorporieren, ob wir sie dabei noch wahrnehmen und auch dem Baum selbst begegnen können, wird abhängen, ob wir - unserer natürlichen Lebensgrundlagen bewusst - eine Zukunft haben.

- Dr. Andreas Losch ist Lehrbeauftragter an der Theologischen Fakultät der Universität Bern. Zu seinen Arbeitsgebieten gehören das jüdisch-christliche Gespräch und der Dialog von Theologie und Naturwissenschaften, insbesondere auf dem Gebiet der Astrobiologie.

\footnotetext{
${ }^{30}$ Ders., Schriften über das dialogische Prinzip, 82.

${ }^{31}$ Vgl. Ders., Schriften zur politischen Philosophie und zur Sozialphilosophie, in: MBW 11 (2019), 129.

32 "...] in dem bewußten Leben kehrt nur welthaftes Sein als menschliches Werden wieder. Der Geist erscheint in der Zeit als Erzeugnis, ja als Nebenprodukt der Natur, und doch ist eben er es, der sie zeitlos umhüllt.« Ders., Schriften über das dialogische Prinzip, 51.

${ }^{33}$ Harald Lesch, Geleitwort, in:Wissenschaft und die Frage nach Gott. Theologie und Naturwissenschaft im Dialog, hg. v. Andreas Losch/Frank Vogelsang, Bonn 2015, 4f.
} 